\title{
CALLIPHORIDAE (DIPTERA) EM MANAUS, AMAZONAS. II. PADRÃO DE ATIVIDADE DE VÔO EM CINCO ESPÉCIES ${ }^{1}$
}

\author{
Norival D. Paraluppi ${ }^{2}$ \\ Elóy G. Castellón ${ }^{3}$
}

\begin{abstract}
CALLIPHORIDAE (DIPTERA) IN MANAUS, AMAZONAS. II. FLIGHT ACTIVITY PATTERN IN FIVE SPECIES. Five species of calliphorid flies, Chrysomya megacephala (Fabricius, 1794), C. putoria (Wiedemann, 1830), C. albiceps (Wiedemann, 1819), Cochliomyia macellaria (Fabricius, 1775) and Phaenicia eximia (Wiedemann, 1819), were collected hourly between $6: 00$ and 18:00h., during five consecutive days per month for 12 moths; using home-made traps baited with rotting bovine lung. The data were statisticaly analyzed with relation to precipitation, temperature, relative humidity and luminosity. The results indicate that precipitation must have acted upon the seasonal abundance of the species, and that these responded equally to temperature, relative humidity and luminosity, throughout the entire day, except for $\mathbf{P}$. eximia, which exhibited greater sensibility to these factors, demonstrating greater activity during the morning hours. The geographic expansion of invader species, associated to the tolerance to climatic variations was briefily discussed.
\end{abstract}

KEY WORDS. Calliphoridae, flies, climatic factors, flight activity

\section{INTRODUÇÃO}

Com a chegada de três espécies de Chrysomya Robineau-Desvoidy, 1830 no Brasil (IMBIRIBA et al., 1977; GUIMARÃES et al., 1978) abriu-se um novo campo de estudos sobre as moscas califorídeas, permitindo aos pesquisadores acompanhar a evolução do processo de colonização por elas desenvolvido em várias regiões invadidas. GUIMARÃES et al. (1979) e PRADO \& GUIMARÃES (1982) discutiram a alta capacidade de dispersão e adaptação exibida pelas espécies invasoras.

$\mathrm{Na}$ cidade de Manaus, Amazonas, as três espécies introduzidas, Chrysomya megacephala (Fabricius, 1794), C. putoria (Wiedemann, 1830) e C. albiceps (Wiedemann, 1819) são muito abundantes, notadamente nos arredores das feiras livres, exibindo alto grau de adaptação na região, cujas condições climática são diferentes daquelas existentes no sul do país, onde também estão bem adaptadas.

1) Parte de Tese de Doutoramento submetida, pelo primeiro autor, ao Programa de Pós-graduação INPA/UFAM.

2) Departamento de Biologia, Universidade Federal do Amazonas, 69077-000 Manaus, Amazonas, Brasil.

3) Instituto Nacional de Pesquisas da Amazônia (INPA), Caixa Postal 478, 69011-970 Manaus, Amazonas, Brasil. 
Desta forma procurou-se, neste trabalho, verificar o grau de influência de alguns fatores climáticos sobre a atividade de vôo das espécies de moscas introduzidas e das endêmicas mais comuns, em função do alto grau de adaptação que aquelas apresentam na região.

\section{MATERIAL E MÉTODOS}

\section{CAPTURA DAS MOSCAS}

Em um local sombreado e aberto foram colocadas duas armadilhas de fabricação caseira, similares àquelas utilizadas por FERREIRA (1978) e LINHARES (1981), tendo como isca pulmão bovino envelhecido durante $36 \mathrm{~h}$, distanciadas cerca de $2,5 \mathrm{~m}$ uma da outra, e suspensas por um varal a $40 \mathrm{~cm}$ do solo, de modo a ficarem sob as mesmas condições ambientais, permanecendo expostas durante cinco dias consecutivos de cada mês, entre 05:30 e 18:00 h, pelo período de 12 meses (setembro de 1988 a agosto de 1989), sendo as iscas substituídas diariamente. A cada hora, a partir das 06:00 h, as moscas foram retiradas das armadilhas, e no laboratório, foram sacrificadas, identificadas, separadas segundo o sexo e contadas, considerando-se conjuntamente o conteúdo das duas armadilhas. As cinco espécies mais abundantes foram utilizadas como material de estudo.

\section{FATORES CLIMÁTICOS}

Simultaneamente à manipulação das armadilhas, a temperatura e a umidade relativa foram medidas no mesmo local com auxílio de um termohigrômetro SUNDO, de ponteiros, instalado à sombra, a $1,5 \mathrm{~m}$ do solo, e a luminosidade, através de um luxímetro digital ICEL-LD-500, com o dispositivo foto-receptor voltado para as armadilhas.

\section{ANÁLISES ESTATÍSTICAS}

Foram aplicadas duas análises de variância não paramétricas (SOKAL \& ROHLF, 1969) para os fatores "mês" e "horário", separadamente para cada uma das cinco espécies de moscas, para se comparar as abundâncias entre os diferentes meses e horários. Para tal foi aplicado um teste de comparação múltipla não paramétrico (SOKAL \& ROHLF, 1969; ZAR, 1974), apenas para aqueles casos mais característicos, tais como: horário de maior incidência de moscas, horário de forte luminosidade (13:00 h), horário crepuscular (17:00 h), meses de maior e menor pluviosidades (maio e agosto, respectivamente), e meses de maior e menor incidência de moscas para cada espécie.

Com a finalidade de se analisar o efeito causado pelos fatores climáticos sobre a atividade das moscas, foram feitos diagramas de dispersão (plots) para fêmeas e machos de cada espécie, contra temperatura, umidade relativa e luminosidade. 


\section{RESULTADOS}

Quando comparados os dados de capturas entre o mês de maior com o de menor densidade pluviométrica, verificou-se através dos resultados, diferenças significativas entre os dois extremos (Tab. I), e isto pode estar evidenciando que as populações de Calliphoridae da região são sensíveis ao fator pluviosidade e, neste caso, diminuem em densidade, ou pelo menos, em atividade de vôo em busca de fontes atrativas, durante a estação chuvosa. A exceção foi Phaenicia eximia (Wiedemann, 1819), cuja freqüência nas armadilhas foi maior no período chuvoso, exibindo portanto, maior densidade populacional ou maior atividade, nesta época.

Tabela I. Teste de comparação não paramét rico para as espécies, em relação aos meses de captura. Para cada espécie estão sendo comparados o mês de maior com o de menor pluviosidade (Mai-Ago), e também o mês de maior com o de menor incidência de moscas.

\begin{tabular}{|c|c|c|c|c|c|c|c|}
\hline Espécies & Meses & Comparação & $\mathbf{P}$ & SE & $\mathrm{R}_{\mathrm{B}}-\mathrm{R}_{\mathrm{A}}$ & $q_{e}$ & $\mathrm{q} 0.01,00, \mathrm{p}$ \\
\hline \multirow[t]{2}{*}{ Chrysomya megacephala } & Mai $x$ Ago & $11 \times 4$ & 8 & 108,766 & $1569,0-648,0=921,0$ & 8,468 & $4,987 *$ \\
\hline & Jul $x$ Fev & $12 \times 1$ & 12 & 162,890 & $1762,5-2805,0=1482,0$ & 9,098 & $5,290 *$ \\
\hline \multirow[t]{2}{*}{ Chrysomya putorla } & Mai $\times$ Ago & $11 \times 4$ & 8 & 108,766 & $1654,0-639,0=1015,0$ & 9,332 & $4,987 *$ \\
\hline & Set $x$ Jun & $12 \times 1$ & 12 & 162,890 & $1695,0-489,0=1206,0$ & 7,404 & $5,290 *$ \\
\hline \multirow[t]{2}{*}{ Chrysomya albiceps } & Mai $x$ Ago & $11 \times 2$ & 10 & 135,828 & $1472,5-409,5=982,0$ & 7,230 & $4,987 *$ \\
\hline & Nov $x$ Jun & $12 \times 1$ & 12 & 162,890 & $1535,5-422,0=1113,5$ & 6,836 & $5,290 *$ \\
\hline \multirow[t]{2}{*}{ Cochllomyla macellaria } & Mai $\times \mathrm{AgO}$ & $9 \times 5$ & 5 & 68,173 & $1290,0-678,5=611,5$ & 8,970 & $4,603 *$ \\
\hline & Out $\times$ Fev & $12 \times 1$ & 12 & 162,890 & $1675,5-454,0=1221,5$ & 7,499 & $5,290 *$ \\
\hline \multirow[t]{2}{*}{ Phaenicia eximla } & Mai $x$ Ago & $8 \times 4$ & 5 & 68,173 & $1257,5 \cdot 717,0=540,5$ & 7,928 & $4,603 *$ \\
\hline & Abr $\times$ Set & $12 \times 1$ & 12 & 162,890 & $1666,5-287,0=1379,5$ & 8,469 & $5,290 *$ \\
\hline
\end{tabular}

* Significativo ao nível de $\mathrm{P}<0,01$.

Quando se comparou os horários de maior freqüência de moscas com o de maior temperatura e luminosidade (13:00 h) e este com um horário crepuscular (17:00 h) (Tab. II), os resultados das análises não foram significativos para Chrysomya megacephala, C. putoria, C. albiceps e Cochliomyia macellaria (Fabricius, 1775), sugerindo que estas espécies foram capturadas com a mesma intensidade ao longo das variações daqueles fatores climáticos, no decorrer do dia todo, e que estas variações não afetam, portanto, a atividade das espécies. Mais uma vez $P$. eximia comportou-se de maneira diferente, exibindo maior atividade nos horários matinais $(07: 00 \times 13: 00 \mathrm{~h}=$ significativo ao nível de $\mathrm{P}<0,01$ ), por volta das 07:00 $\mathrm{h}$.

As figuras 1 e 2 representam os diagramas de dispersão para fêmeas de C. megacephala e de $P$. eximia, contra temperatura, umidade relativa e luminosidade, onde, comparativamente, são verificadas as diferenças de atividade destas espécies em relação aos fatores climáticos, no decorrer do dia. Os machos tiveram comportamento semelhante ao das fêmeas de suas respectivas espécies. Através da figura 1 observa-se que a distribuição das freqüências de C. megacephala nas armadilhas foi homogênea ao longo das variações de temperatura, umidade relativa e luminosidade, demonstrando assim, que esta espécie exibiu 

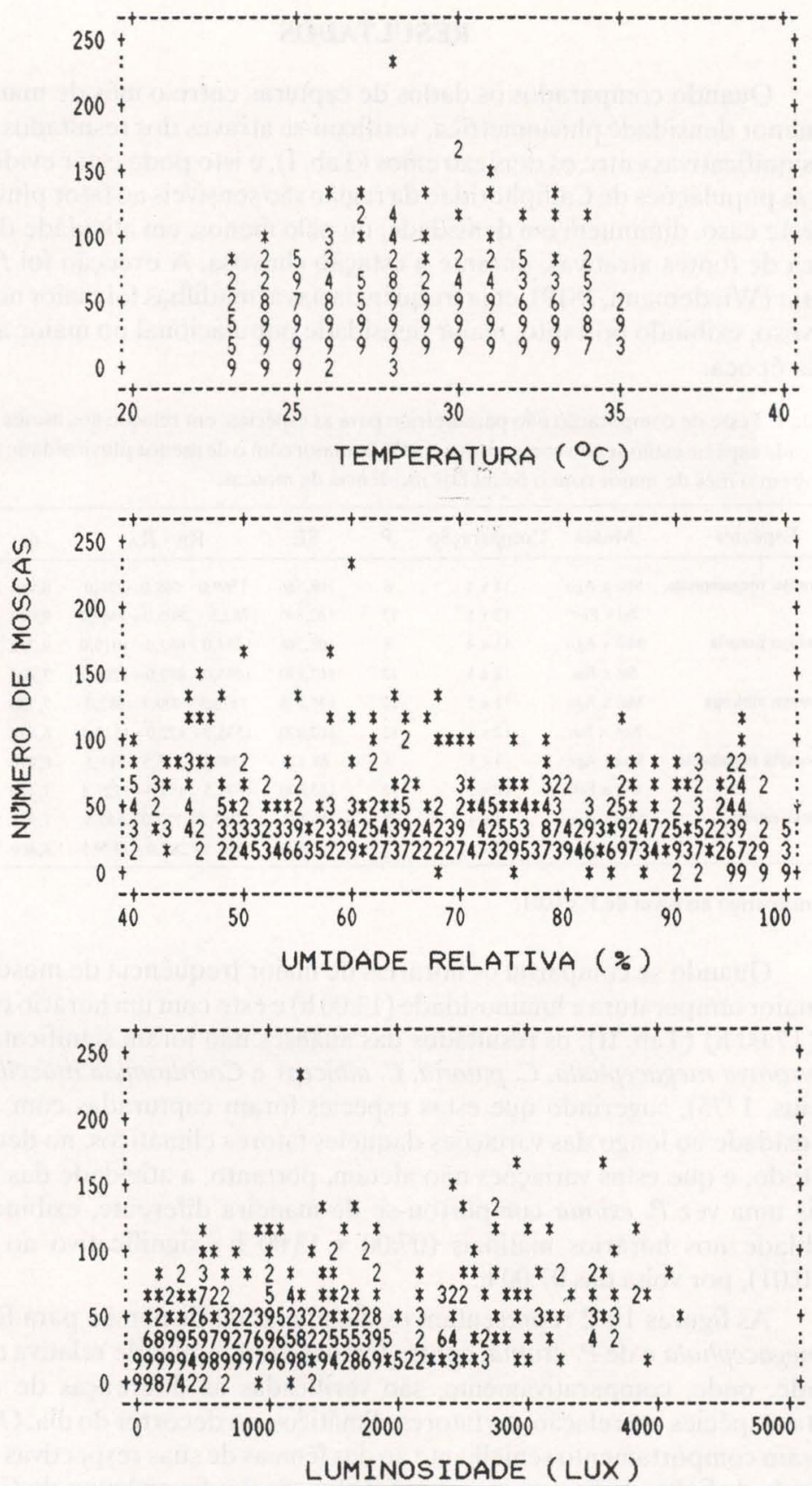

Fig. 1. Diagrama de dispersão representando a distribuição da abundância das fêmeas de C. megacephala no decorrer do dia, em relação à temperatura, umidade e luminosidade. 
Tabela II. Teste de comparação não paramétrico para as espécies, em relação aos horários. Para cada espécie estão sendo comparados os horários de maior incidência de moscas, com o de maior luminosidade e temperatura e este, com um horário de menor luminosidade e temperatura.

\begin{tabular}{|c|c|c|c|c|c|c|c|}
\hline Espécies & Horários & Comparação & $\mathrm{P}$ & SE & $\mathbf{R}_{\mathrm{B}}-\mathrm{R}_{\mathrm{A}}$ & $\mathrm{qe}_{\mathrm{e}}$ & $\mathrm{q} 0.01,00, \mathrm{p}$ \\
\hline \multirow[t]{2}{*}{ Chrysomya megacephala } & $08: 00 \times 13: 00$ & $13 \times 3$ & 11 & 132,499 & $1284,5-878,5=406,0$ & 3,064 & 5,227 \\
\hline & $13: 00 \times 17: 00$ & $7 \times 3$ & 5 & 60,498 & $980,0-878,5=101,5$ & 1,678 & 4,600 \\
\hline \multirow[t]{2}{*}{ Chrysomya putoria } & $08: 00 \times 13: 00$ & $6 \times 4$ & 3 & 36,497 & $1010,0-971,0=39,0$ & 1,069 & 4,120 \\
\hline & $13: 00 \times 17: 00$ & $6 \times 5$ & 2 & 24,495 & $1010,0-976,0=34,0$ & 1,388 & 3,643 \\
\hline \multirow[t]{2}{*}{ Chrysomya albiceps } & $10: 00 \times 13: 00$ & $13 \times 8$ & 8 & 96,499 & $1258,0-1098,0=160,0$ & 1,658 & 4,987 \\
\hline & $13: 00 \times 17: 00$ & $8 \times 5$ & 4 & 48,497 & $1098,0-966,5=131,5$ & 2,712 & 4,403 \\
\hline \multirow[t]{2}{*}{ Cochliomyla macellaria } & $10: 00 \times 13: 00$ & $13 \times 4$ & 10 & 120,499 & $1195,0-891,0=304,0$ & 2,523 & 5,157 \\
\hline & $13: 00 \times 17: 00$ & $7 \times 4$ & 4 & 48,497 & $1003,5-891,0=112,5$ & 2,320 & 4,403 \\
\hline \multirow[t]{2}{*}{ Phaenicla eximia } & $07: 00 \times 13: 00$ & $13 \times 4$ & 10 & 120,499 & $1608,5 \cdot 714,0=894,5$ & 7,423 & $5,157 *$ \\
\hline & $13: 00 \times 17: 00$ & $6 \times 4$ & 3 & 36.497 & $814,5-714,0=100,5$ & 2.754 & 4,120 \\
\hline
\end{tabular}

* Significativo ao nível de $\mathrm{P}<0,01$.

atividade constante durante as horas do dia. Quanto à $P$. eximia, a maior distribuição das freqüências para temperaturas e luminosidades mais amenas e umidades mais elevadas (Fig. 2), demonstra que esta espécie exibiu maior atividade nos horários matinais.

Os diagramas de dispersão referentes às demais espécies não estão representados, mas os resultados para ambos os sexos foram semelhantes aqueles observados para C. megacephala.

Assim, verifica-se que, com exceção de P. eximia, todas as demais espécies não apresentam nenhuma tendência para o aumento ou diminuição de suas freqüências em relação às três variáveis ambientais consideradas, em qualquer hora do dia. Para P. eximia, no entanto, de modo não linear, tanto que, para temperaturas baixas, a variância da abundância foi maior que para temperaturas altas. Este fenômeno também ocorreu em relação à luminosidade. Quanto à umidade relativa, a abundância de indivíduos capturados foi maior para as umidades mais elevadas.

\section{DISCUSSÃO}

Procurou-se estudar a influência dos fatores climáticos sobre a atividade de vôo das moscas califorídeas, em relação à busca pela fonte atrativa, quer seja à procura de alimento, de substrato para oviposição, ou de ambos, ficando demonstrado que as três espécies invasoras de Chrysomya e a espécie endêmica do Novo Mundo, Cochliomyia macellaria, exibem comportamentos semelhantes no decorrer do dia. Esta semelhança de comportamentos pode ser um dos fatores a estar favorecendo as espécies invasoras na ocupação do mesmo nicho da endêmica, deslocando-a, como já foi observado por outros autores, em várias regiōes (GUIMARÃES et al., 1979; FERREIRA, 1983; BAUM- GARTNER \& GREENBERG, 1984).

Phaenicia eximia, no entanto, outra espécie endêmica do Novo Mundo, 

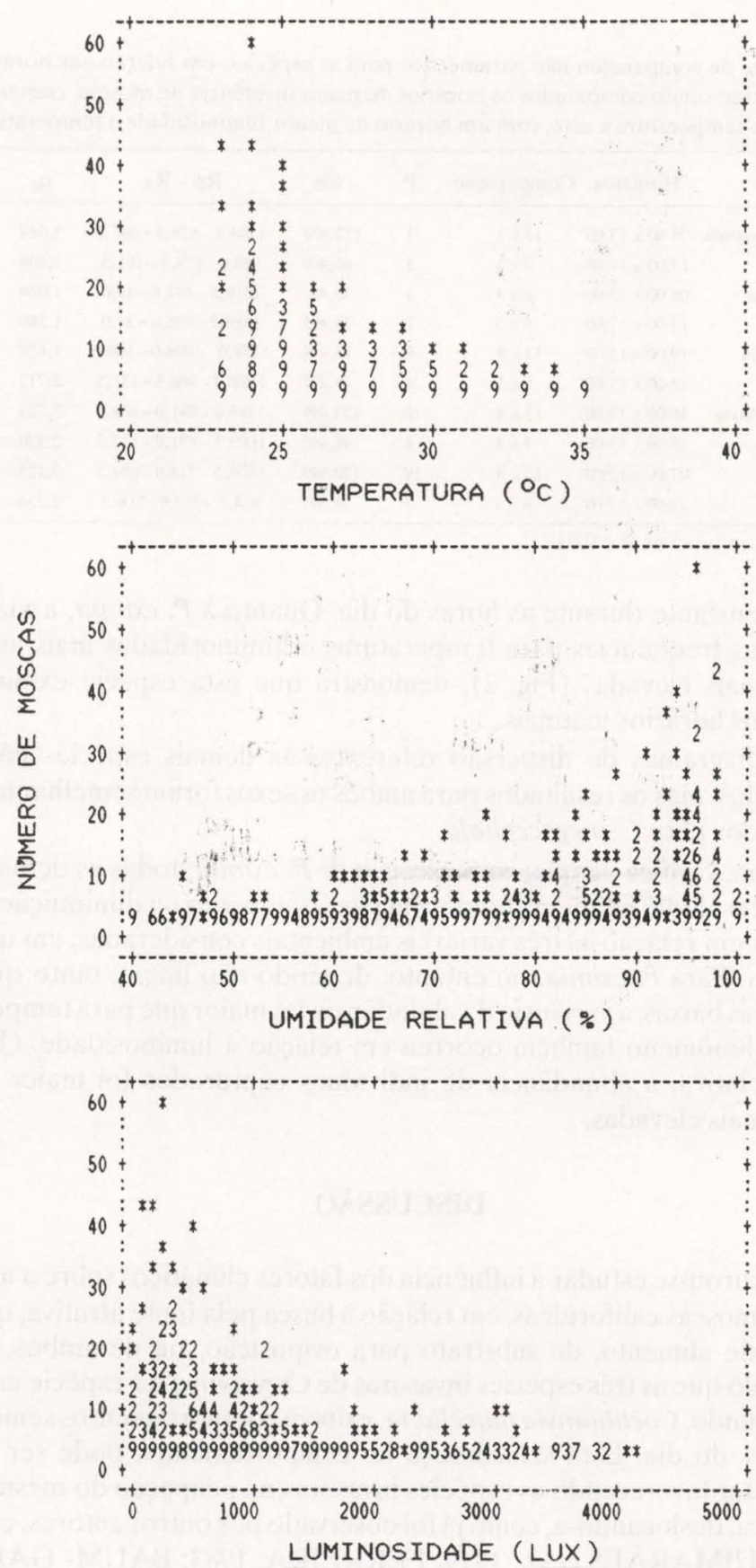

Fig. 2. Diagrama de dispersão representando a distribuição da abundância das fêmeas de P. eximia no decorrer do dia, em relação à temperatura, umidade relativa e luminosidade. 
exibiu maior sensibilidade, provavelmente à temperatura. NICHOLSON (1934), VOGT et al. (1983) e VOGT (1988) verificaram na Austrália, que este foi o fator que mais interferiu nas capturas de califorídeos, embora o primeiro autor considere que existem fatores complexos atuando sobre a relação entre a atividade e temperatura. Assim, o fato desta espécie ter sido principalmente ativa no período matinal, de menor temperatura, pode ser um reflexo de sua atividade de oviposição. AVANCINI (1986) e AVANCINI \& LINHARES (1988) verificaram que esta espécie entrava nas armadilhas principalmente para ovipositar ao invés de para se alimentar, quando a isca utilizada era carcaça de camundongo.

Por outro lado, em Manaus a amplitude da variação da temperatura diária tende a ser elevada, atingindo mais de $10^{\circ} \mathrm{C}$ entre as primeiras horas matinais e o crepúsculo. Desta forma, pode-se também considerar que $P$. eximia não conseguiria manter a mesma freqüência de atividade de vôo durante os horários mais quentes, devido à adaptação adquirida a partir dos efeitos provenientes das condições ambientais previamente experimentadas durante os horários de menor temperatura, estando de acordo com as observações de DIGBY (1958), para Calliphora erythrocephala (Meigen, 1826).

A tolerância às variações climáticas, como temperatura, umidade relativa e luminosidade exibida pelas espécies invasoras, pode ser considerada como um dos fatores determinantes da alta capacidade adaptativa verificada para estas espécies nas regiões invadidas, facilitando assim, a expansão geográfica a que elas vêm se submetendo.

\section{REFERÊNCIAS BIBLIOGRÁFICAS}

AVANCINI, R.M.P. 1986. Fases de desenvolvimento ovariano em seis espécies de Calliphoridae (Diptera). Revta bras. Ent. 30 (2): 359-364.

AVANCINI, R.M.P. \& A.X. LINHARES. 1988. Selective attractiveness of rodent-baited traps for female blowflies. Med. and Vet. Entomol. 2: 73-76.

BAUMGARTNER, D.L. \& B. GREENBERG. 9184. The genus Chrysomya (Diptera: Calliphoridae) in the New World. J. Med. Entomol. 21 (1): 105113.

DIGBY, P.S.B. 1958. Flight activity in the blowfly Calliphora erythrocephala, in relation to light and radiant heat, with special reference to adaptation. J. Exp. Biol. 35 (1): 1-19.

FERREIRA, M.J. DE M. 1978. Sinantropia de dípteros muscóideos de Curitiba, Paraná. I. Calliphoridae. Rev. Brasil. Biol. 38 (2): 445-454.

. 1983. Sinantropia de Calliphoridae (Diptera) em Goiânia, Goiás. Rev. Brasil. Biol. 43 (2): 199-210.

GUIMARÃES, J.H.; A.P. PRADO \& A.X. LINHARES. 1978. Three newly introduced blowfly species in southern Brazil (Diptera: Calliphoridae). Revta bras. Ent. 22 (1): 53-60.

GUIMARÃES, J.H.; A.P. PRADO \& G.M. BURALLI. 1979. Dispersal and distribution of three newly introduced species of Chrysomya Robineau- 
Desvoidy in Brazil (Diptera: Calliphoridae). Revta bras. Ent. 23 (4): 245-255. IMBIRIBA, A.S.; D.T. IZUTANI; I.T. MILHORETTO \& E. LUZ. 1977. Introdução de Chrysomya chloropyga (Wiedemann, 1818) na Região Neotropical (Diptera: Calliphoridae). Arq. Biol. Tecnol. 20: 35-39.

LINHARES, A.X. 1981. Synanthropy of Calliphoridae and Sarcophagidae (Diptera) in the city of Campinas, São Paulo, Brazil. Revta bras. Ent. 25 (3): 189-215.

NICHOLSON, A.J. 1934. The influence of temperature on the activity of sheep-blowflies. Bull. ent. Res. 25: 85-99.

PRADO, A.P. \& J.H. GUIMARÃES. 1982. Estado atual da distribuição e dispersão das espécies do gênero Chrysomya R-D na região Neotropical (Diptera, Calliphoridae). Revta bras. Ent. 26 (3-4): 225-231.

SOKAL, R.R. \& F.J. ROHLF. 1969. Biometry. The principles and practice of statistics in biological research. San Francisco, W.H. Freeman and Company, 776p.

VOGT, W.G. 1988. Influences of weather on trap catches of Chrysomya rufifacies (Diptera: Calliphoridae). J. Aust. Entomol. Soc. 27 (2): 99-103.

VOGT, W.G.; T.L. WOODBURN; R. MORTON \& B.A. ELLEN. 1983. The analysis and standardisation of trap catches of Lucilia cuprina (Wiedemann) (Diptera: Calliphoridae). Bull. ent. Res. 73 (4): 609-617.

ZAR, J.H. 1974. Biostatistical Analysis. Englewood Cliffs, Prentice-Hall Inc., $620 \mathrm{p}$.

Recebido em 20.X.1993; aceito em 24.III.1994. 